Article

\title{
Quasi-Static Elastography and Ultrasound Plane-Wave Imaging: The Effect of Beam-Forming Strategies on the Accuracy of Displacement Estimations
}

\author{
Gijs A.G.M. Hendriks ${ }^{1, *(D)}$, Chuan Chen ${ }^{1}$, Hendrik H.G. Hansen ${ }^{1}$ and Chris L. de Korte ${ }^{1,2}$ (D) \\ 1 Medical UltraSound Imaging Center, Department of Radiology and Nuclear Medicine, Radboud University \\ Medical Center, P.O. Box 9101, 6500 HB Nijmegen, The Netherlands; chuan.chen@radboudumc.nl (C.C.); \\ rik.hansen@radboudumc.nl (H.H.G.H.); chris.dekorte@radboudumc.nl (C.L.d.K.) \\ 2 Physics of Fluids Group, MIRA, University of Twente, P.O. Box 217, 7500 AE Enschede, The Netherlands \\ * Correspondence: gijs.hendriks@radboudumc.nl; Tel.: +31-24-365-1503
}

Received: 22 December 2017; Accepted: 17 February 2018; Published: 26 February 2018

\begin{abstract}
Quasi-static elastography is an ultrasound method which is widely used to assess displacements and strain in tissue by correlating ultrasound data at different levels of deformation. Ultrafast plane-wave imaging allows us to obtain ultrasound data at frame rates over $10 \mathrm{kHz}$, permitting the quantification and visualization of fast deformations. Currently, mainly three beam-forming strategies are used to reconstruct radio frequency (RF) data from plane-wave acquisitions: delay-and-sum (DaS), and Lu's-fk and Stolt's-fk operating in the temporal-spatial and Fourier spaces, respectively. However, the effect of these strategies on elastography is unknown. This study investigates the effect of these beam-forming strategies on the accuracy of displacement estimation in four transducers (L7-4, 12L4VF, L12-5, MS250) for various reconstruction line densities and apodization/filtering settings. A method was developed to assess the accuracy experimentally using displacement gradients obtained in a rotating phantom. A line density with multiple lines per pitch resulted in increased accuracy compared to one line per pitch for all transducers and strategies. The impact on displacement accuracy of apodization/filtering varied per transducer. Overall, Lu's-fk beam-forming resulted in the most accurate displacement estimates. Although DaS in some cases provided similar results, Lu's-fk is more computationally efficient, leading to the conclusion that Lu's-fk is most optimal for plane wave ultrasound-based elastography.
\end{abstract}

Keywords: quasi-static; elastography; ultrasound; beam-forming; delay-and-sum; Stolt's; Lu's; displacements; apodization; lateral displacement; axial displacement

\section{Introduction}

In 1991, Ophir et al. [1] proposed a new ultrasound technique, quasi-static elastography, to visualize and quantify deformation of tissue to thus detect abnormalities (e.g., through the relatively stiffer breast and prostate tumors). In this technique, ultrasound radio frequency (RF) data are obtained prior to and after deformation of the target tissue. Deformation can be induced externally by the transducer or a vibrator, or internally by the heart or respiration. Several methods have been developed to estimate displacement or strain maps based on the acquired RF data [2-6]. Conventionally, a template windows containing post-deformation RF-data is cross-correlated with a search window containing pre-deformation data. The position of the cross-correlation peak indicates the displacement of the template. To cope with relatively large displacements, coarse-to-fine cross-correlation methods were developed [2]. In coarse-to-fine cross-correlation, multi-iterative cross-correlation is executed 
starting with relatively large windows analyzing the envelope signal and subsequently using finer windows of RF-signal in each subsequent iteration with the estimated displacements of the previous iteration as an offset. Finally, a least squares strain estimator can be used to reconstruct a strain map [7].

The majority of current commercial ultrasound systems collect ultrasound RF data line-by-line using focused ultrasound transmit-and-receive sequences. Back in 1979, plane wave acquisitions were proposed as alternative to line-by-line sequences $[8,9]$. This type of acquisition has been getting more attention since the mid-2000s when the computational power and data transfer rates had sufficiently increased to enable the processing of the data generated by plane-wave transmissions. In plane-wave imaging, the full transducer array is used to transmit one unfocussed plane-wave and receive the reflected signals (Figure 1). In case of a 192-element array transducer, frame-rates can be increased by a factor of 192 compared to line-by-line scans. Consequently, ultrasound data can be collected with frame-rates over $10 \mathrm{kHz}$ (theoretically limited by the two-way propagation of the ultrasound signal) and so fast deformations can also be quantified and visualized using quasi-static elastography. In the absence of focusing in transmit, plane-wave imaging requires software beam-forming to reconstruct RF-data from the obtained element data. In conventional ultrasound scanners, beam-forming is often applied in hardware. In this study, three software beam-forming strategies and their effect on displacement estimation were exploited: delay-and-sum (DaS) [10] in the spatial-temporal domain originating from conventional focused ultrasound, and $\mathrm{Lu}^{\prime} \mathrm{s}-\mathrm{fk}[11,12]$ and Stolt's-fk [13] beam-forming methods, both operating in the Fourier domain which are specifically developed for plane-wave acquisitions.

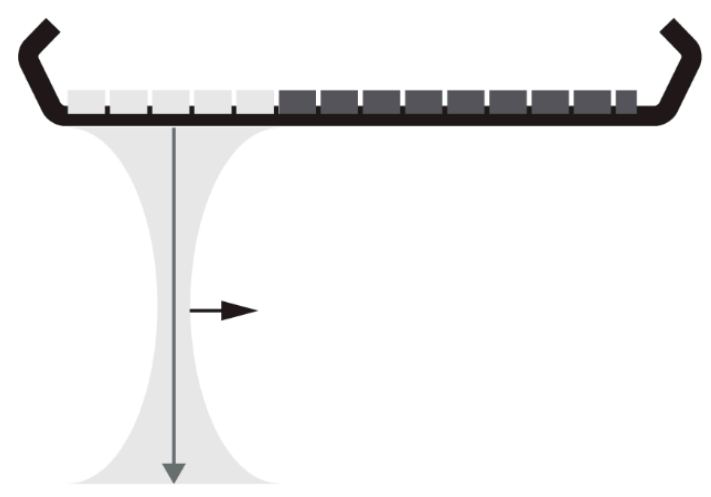

(a)

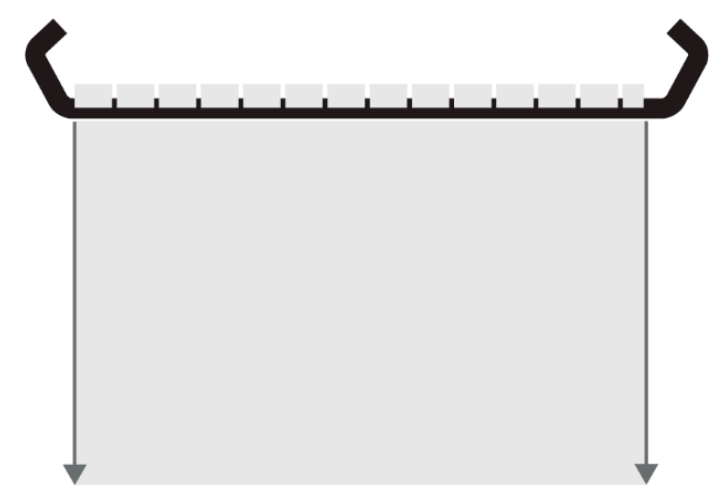

(b)

Figure 1. Illustrations of: (a) a focused acquisition series in which the aperture (active elements in light gray) is shifted to acquire data line-by-line by transmitting a focused ultrasound beam and receiving the reflected signal for each aperture position; (b) a plane-wave acquisition, where an unfocused ultrasound is transmitted and reflected signals are received by the full transducer aperture.

DaS is a beam-forming method based on delaying the element signal by the expected time-of-flight (ToF) and summation of the element data points (Figure 2). For a certain point to be reconstructed, the ToF is calculated as the time of the plane-wave propagating to that point and the reflected or back-scattered signal to be received by the elements. The ToF is used to identify and sum the signals originating from each point in the element data. In dynamic focusing in receive, the F-number (F) dictates the number of elements (aperture) used for beam-forming:

$$
F=\frac{d}{l_{a p p}}
$$

where $d$ and $l_{\text {app }}$ are the depth of the reconstruction point and aperture width, respectively. Furthermore, apodization can be applied to weigh the signals (e.g., by Hamm or Hann function) to reconstruct 
each point and so expected angle sensitivity and back-scatter signal intensities can be incorporated. Apodization is often applied to increase contrast in B-mode images.

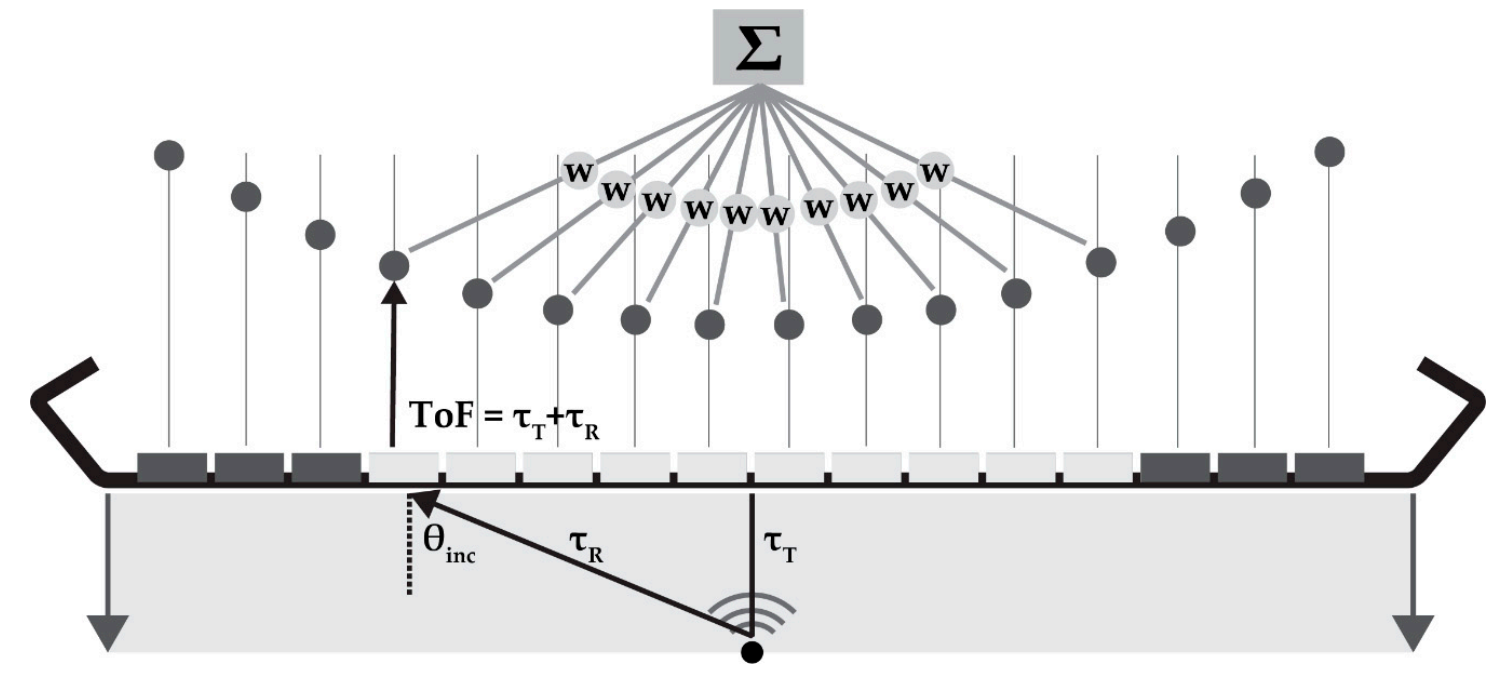

Figure 2. Schematic overview of delay-and-sum (DaS) beam-forming: for each reconstruction point, the time-of-flight $(\mathrm{ToF})$ can be calculated by the transmit $\left(\tau_{\mathrm{T}}\right)$ and receive time towards each element $\left(\tau_{R}\right)$. These ToF values are used to delay and sum the element data; if required the data can be weighted (w) by an apodization function. $\theta_{\text {inc }}$ is the incidence angle of the signal.

Another approach is beam-forming in the Fourier domain by Lu's-fk or Stolt's-fk which are computationally less expensive and can run around 25 times faster compared to DaS [13]. In both methods, 2-D element data in the spatial-temporal domain are transformed to the fk-space. Next, a migration method is applied to transform the data into the complete k-space and finally the migrated data are transformed back into the spatial-temporal domain (Figure 3). A migration method of plane-wave ultrasound was first carried out by $\mathrm{Lu}$ et al. from the analysis of limited diffraction beams [11,12]. Lu et al. introduced the mapping rule throughout the transmission-scattering-receiving process. More recently, Garcia et al. proposed another method by adapting Stolt's-fk seismic wave migration to the plane wave ultrasound scenario [13]. In this modification, the plane wave's backscattering is fitted with waves spontaneously radiating from sources. Comparing Stolt's-fk and Lu's-fk, the two methods share a similar spectrum content at small angles, while their spectra differ more significantly in the mapping of large angles.

Similar to apodization in DaS, angular weighting can be applied in Fourier domain beam-forming to increase contrast in the final B-mode image [14]. In this method, developed in our group, the Fourier spectrum after the migration (k-space) is multiplied with a template which is designed such that reflected waves originating from directions closely aligned with the plane-wave steering angle pass unaltered, whereas waves from wave directions deviating from the plane-wave propagation direction are attenuated according to a Hann function (Figure 3). This template can be applied for both migration methods (Lu's-fk and Stolt's-fk).

Since the beam-forming in plane-wave imaging is executed using software, the design of the beam-forming reconstruction grid (USGrid) is more flexible compared to conventional focused line-by-line scanners in which beam-forming is executed by hardware. Consequently, more lines per pitch can be reconstructed, which might result in more accurate displacement estimates. For focused imaging, it has already been shown that more accurate lateral displacement estimates can be obtained when increasing the RF line density by interpolation [15,16].

Plane-wave imaging and displacement and strain imaging have been combined in multiple studies. Besides a paper from our group [17] which investigated the effect of line density on displacement 
estimation for DaS, to our knowledge, the effects of different beam-forming strategies and line densities on displacement and strain estimation have not been explored. Therefore, the aim of this study was to investigate the effect of beam-forming strategies (DaS, Lu's-fk and Stolt's-fk) for plane-wave imaging on the accuracy of displacement estimates. Furthermore, the effect of the line density, apodization (in $\mathrm{DaS}$ ) and angular weighting (in Lu's-fk and Stolt's-fk) were evaluated, and four different transducers were used with central frequencies varying between 5 and $21 \mathrm{MHz}$ representing the whole range of clinically used linear array transducers. In this study, we evaluated the performance of displacement and strain estimation by using a rotating phantom. The advantage of a rotating phantom is that a displacement field is induced with axial and lateral displacements varying separately in magnitude instead of having pure axial or lateral displacements with same magnitude as obtained by linear translation of a phantom. Furthermore, the gradients of the axial and lateral displacement field were used to evaluate the accuracy of the displacement estimations because these gradients should be zero (see Materials and Methods section) without requiring exact knowledge of the reference displacement field. These gradients can also be considered as strains since strains are calculated the same way and are equal to their gradient.

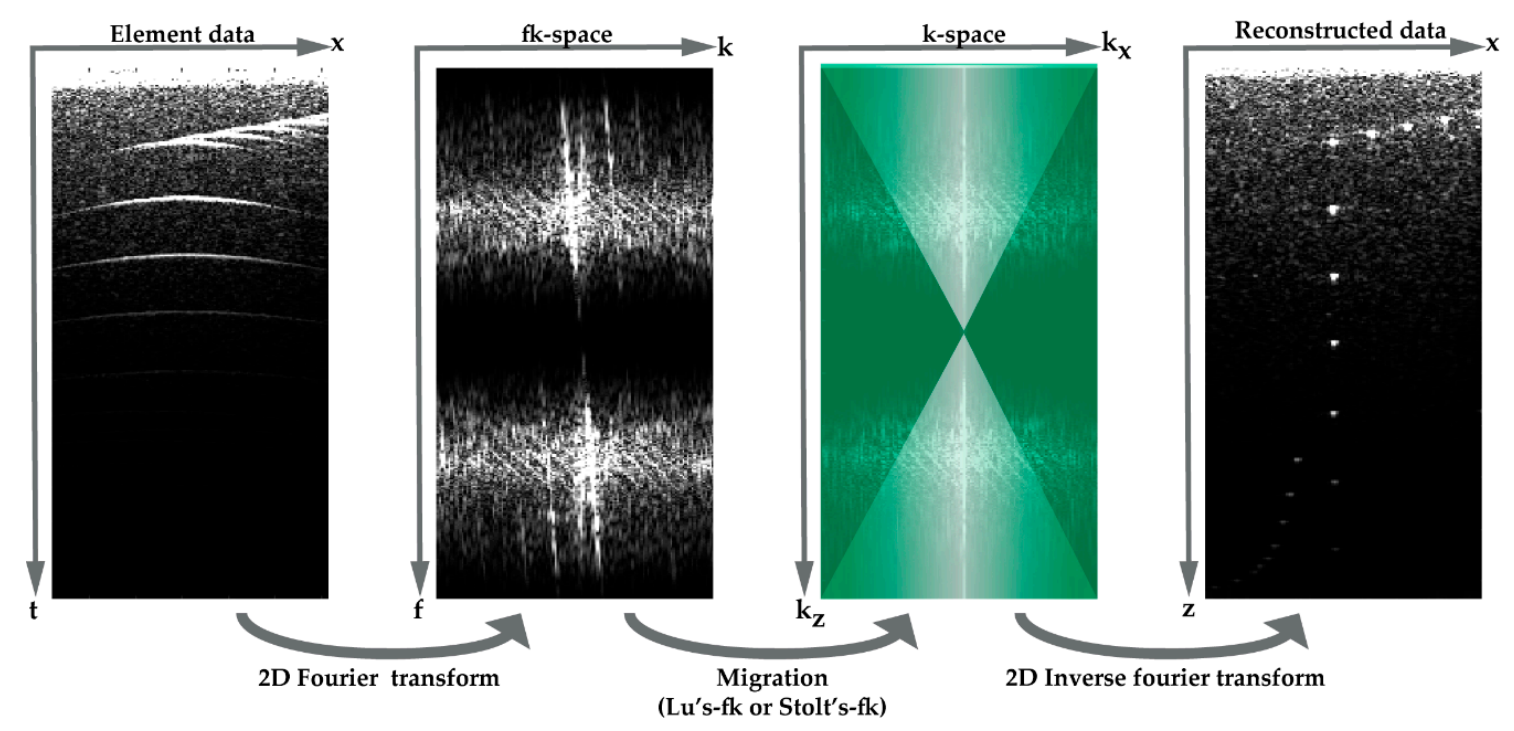

Figure 3. Overview of beam-forming in the fk-space: acquired element data is transformed to the fk-space using 2-D Fourier transform, and Lu's-fk or Stolt's-fk migration is applied to convert the data into the k-space. If required, this spectrum can be multiplied by a template (green overlay) to filter the data. Finally, the reconstructed data can be obtained by the 2-D inverse Fourier transform.

\section{Materials and Methods}

To create a block phantom, gelatin ( $10 \%$ by weight; VMR International, Leuven, Belgium) was dissolved in demineralized water and heated to $90{ }^{\circ} \mathrm{C}$ and cooled to $35^{\circ} \mathrm{C}$ while continuously stirring using a magnetic stirrer. During the cooling process, silica particles ( $2 \%$ by weight; silica gel 60 , 0.015-0.040 mm; Merck KGaA, Darmstadt, Germany) were added, which act as scatterers. Next, the mixed solution was poured in an open container $(200 \times 100 \times 100 \mathrm{~mm})$ leaving a $20 \mathrm{~mm}$ space under the top surface. Finally, the phantom was placed in a fridge to consolidate for $24 \mathrm{~h}$.

The phantom, including the container, was positioned on top of a seesaw to enable rotation of the phantom. One of the four different linear array transducers utilized in this study (Table 1) was positioned above the phantom top surface such that the transducer footprint was unable to touch the phantom after rotation at the maximum angle of 10 degrees. Water was poured on the top surface of the phantom to fill the remaining $20 \mathrm{~mm}$ space and to ensure acoustic coupling between the transducer and phantom. The transducer was connected to a Verasonics V1 system (MS250 to Verasonics Vantage) 
to enable $0^{\circ}$ plane-wave transmissions and element data collection using the center 128 elements of the transducer (MS250: all 256 elements). Element data were recorded prior to and after rotation $\left(\sim 2^{\circ}\right)$ of the phantom. The experimental setup is also visualized in Figure 4.

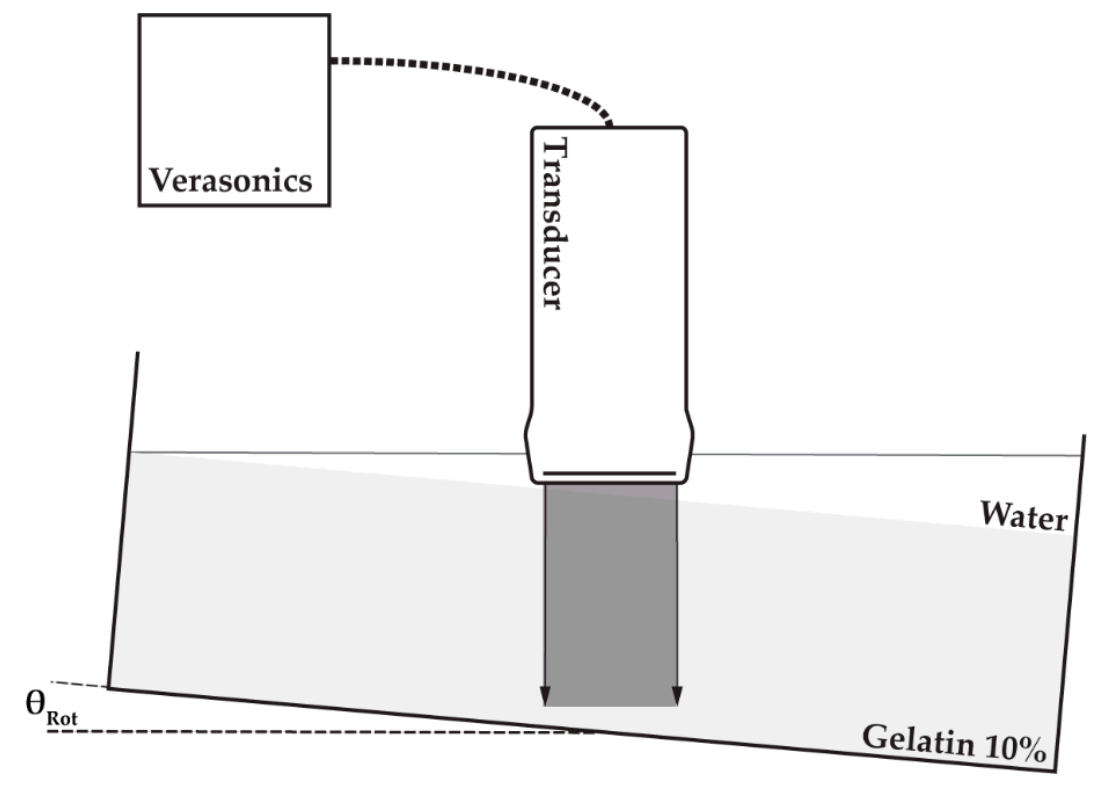

Figure 4. Experimental setup: transducer (Table 1) was connected to a Verasonics (V1 or Vantage) research ultrasound machine; element data were acquired prior to and after rotation $\left(\theta_{\text {Rot }}\right)$ of a container in which a gelatin phantom was positioned (light grey) and water was poured on top of the phantom to ensure ultrasonic coupling.

Table 1. Transducers used in this study with their bandwidth, central frequency $\left(f_{c}\right)$, and pitch.

\begin{tabular}{ccccc}
\hline Transducer & Bandwidth & $\mathbf{f}_{\mathbf{c}}$ & Pitch & Manufacturer \\
\hline L7-4 & $4-7 \mathrm{MHz}$ & $5.0 \mathrm{MHz}$ & $298 \mu \mathrm{m}$ & ATL $^{1}$ \\
$12 \mathrm{~L} 4 \mathrm{VF}$ & $4-12 \mathrm{MHz}$ & $8.2 \mathrm{MHz}$ & $266 \mu \mathrm{m}$ & Siemens $^{2}$ \\
L12-5 & $5-12 \mathrm{MHz}$ & $9.0 \mathrm{MHz}$ & $198 \mu \mathrm{m}$ & ATL $^{1}$ \\
MS250 & $13-24 \mathrm{MHz}$ & $21 \mathrm{MHz}$ & $88 \mu \mathrm{m}$ & VisualSonics $^{3}$ \\
\hline
\end{tabular}

${ }^{1}$ ATL, Bothell, WA, USA; ${ }^{2}$ Siemens Healthineers, Issaquah, WA, USA; ${ }^{3}$ FUJIFILM VisualSonics Inc., Toronto, ON, Canada.

Element data were beam-formed using three different strategies: (1) Lu's-fk [11,12], Stolt's-fk [13], and DaS [10]; (2) with and without angular weighting (Lu's-fk; Stolts-fk) [14] or apodization by Hamm function (DaS); and (3) a beam-forming ultrasound grid (USGrid) with 8 samples per wave length and 1, 2, 3 and 4 lines per pitch. These strategies were implemented in Matlab (2016a; Mathworks Inc., Natick, MA, USA). Zero-padding was applied in the k-space to achieve higher line-densities for the fk-based strategies. Beam-forming settings were empirically determined: plane-wave element data were acquired in a multi-purpose phantom and beam-forming settings were tuned resulting in optimal contrast and resolution after beam-forming (see Appendix A for more details). For all transducers, the F-number ( $\mathrm{DaS}$ ) and angle weighting range (Lu's-fk; Stolts-fk) were set to 0.875 and $\pm 20^{\circ}$, respectively.

Next, displacements were estimated by two step coarse-to-fine normalized cross correlation of pre- and post-rotation beam-formed ultrasound envelope and RF data in the first and second step respectively [18,19]. The cross-correlation peak was interpolated (2-D spline) after the final iteration to estimate sub-sample displacements. After each iteration, displacements were median filtered to remove outliers. Template and search windows, and other settings can be found in Table 2. 
Table 2. Settings of the displacement estimation algorithm and displacement grid (DispGrid).

\begin{tabular}{cccccc}
\hline Transducer & Step & Template Window $^{\mathbf{1}}$ & Search Window $^{\mathbf{1}}$ & Filter Size $^{2}$ & DispGrid $^{\mathbf{1}}$ \\
\hline L7-4 & 1 & $1.22 \times 1.49$ & $4.82 \times 5.07$ & $5 \times 5$ & $0.298 \times 0.298$ \\
- & 2 & $0.62 \times 0.90$ & $0.89 \times 1.49$ & $3 \times 3$ & $0.298 \times 0.298$ \\
12L4VF & 1 & $0.68 \times 1.33$ & $2.68 \times 4.52$ & $5 \times 5$ & $0.266 \times 0.266$ \\
- & 2 & $0.35 \times 0.80$ & $0.49 \times 1.33$ & $3 \times 3$ & $0.266 \times 0.266$ \\
L12-5 & 1 & $0.68 \times 0.99$ & $2.68 \times 3.37$ & $5 \times 5$ & $0.198 \times 0.198$ \\
- & 2 & $0.35 \times 0.60$ & $0.49 \times 0.99$ & $3 \times 3$ & $0.198 \times 0.198$ \\
MS250 & 1 & $0.29 \times 0.44$ & $1.15 \times 1.50$ & $5 \times 5$ & $0.088 \times 0.088$ \\
- & 2 & $0.15 \times 0.27$ & $0.21 \times 0.44$ & $3 \times 3$ & $0.088 \times 0.088$ \\
\hline
\end{tabular}

${ }^{1}$ Axial $\times$ lateral window size or DispGrid resolution in $\mathrm{mm} ;{ }^{2}$ \# samples and \# lines in DispGrid which is independent of \# lines per pitch.

The axial and lateral displacements $\left(u_{z}\right.$ and $\left.u_{x}\right)$ after rotation can be described as:

$$
\begin{aligned}
& u_{x}=(\cos (\theta)-1)\left(x-x_{0}\right)-\sin (\theta)\left(z-z_{0}\right) \\
& u_{z}=\sin (\theta)\left(x-x_{0}\right)+(\cos (\theta)-1)\left(z-z_{0}\right)
\end{aligned}
$$

where $\theta$ and $\left(x_{0}, z_{0}\right)$ are the rotation angle and center coordinates, respectively. The gradient of $u_{x}$ and $u_{z}$ in the lateral $(x)$ and the axial $(z)$ directions can respectively be described as:

$$
\begin{aligned}
& s_{x x}=\frac{d u_{x}}{d x}=\cos (\theta)-1 \\
& s_{z z}=\frac{d u_{z}}{d z}=\cos (\theta)-1
\end{aligned}
$$

In this experiment, angle $\theta$ can be considered small $\left(\sim 2^{\circ}\right)$ and so it can be assumed that:

$$
\cos (\theta) \approx 1-\frac{\theta^{2}}{2} \approx 1
$$

Consequently, $s_{x x}$ and $s_{z z}$ in (Equations (4) and (5)) approximate zero, independently of the rotation angle and center. As these gradients (strains) yield 0 and the exact rotational angle and center were unknown in this experiment, we adopted the root-mean squared error (RMSE) of the gradients as a measure of the accuracy of the displacement estimates. The gradients of the displacements were calculated using a one-dimensional three-point least-squares strain estimator [7]. Gradients were only calculated and evaluated within a field-of-view (FoV) measuring -10 to $10 \mathrm{~mm}$ laterally and 17.5 to $52.5 \mathrm{~mm}$ axially for all transducers. However, the FoV measurement of the MS250 transducer was -8 to $8 \mathrm{~mm}$ laterally, since the FoV was limited by the transducer footprint. The top 17.5-mm axial depth was measured through water and was therefore neglected for all transducers. All acquisition and processing steps and intermediate results are summarized in Figure 5. 


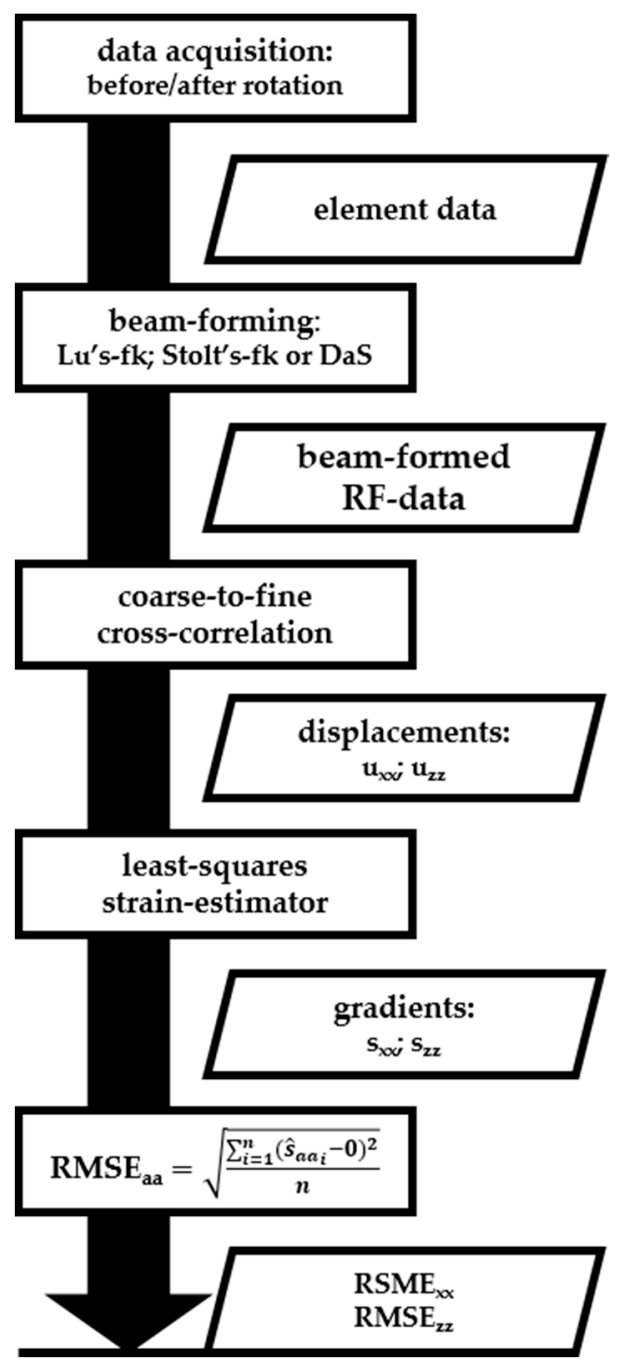

Figure 5. Summary of the processing flow described in the Material and Methods section. This work flow was repeated for every transducer (Table 1). Rectangles represent processing steps (acquisitions or calculations) and parallelograms represent (intermediate) results.

\section{Results}

Figure 6 provides an overview of the estimated axial and lateral displacement and strain fields for all probes using Lu's-fk beam-forming and a line density of two lines per pitch. As expected from Equations (2) to (6), the axial displacement fields (Figure 6a-d) were constant in the axial direction and so the gradient of the axial displacements in axial directions $\left(s_{z z}\right)$ were approximately $0 \%$ (see Figure $6 \mathrm{f}-\mathrm{i}$ ). For the 12L4VF transducer the results revealed some artifacts in the left top corner (Figure $6 \mathrm{~b}, \mathrm{~g}$ ). These artifacts were visible for all beam-forming strategies and line densities. The MS250 transducer showed more outliers at lower depths $(30-52.5 \mathrm{~mm})$ which were probably caused by attenuation of the ultrasound signal at relatively large depths for this frequency.

The lateral displacement (Figure $6 \mathrm{k}-\mathrm{n}$ ) and strain fields (Figure $6 \mathrm{p}-\mathrm{s}$ ) resulted in similar observation as the axial results: constant displacement values in lateral direction and so approximately $0 \%$ gradient of lateral displacements in that direction $\left(s_{x x}\right)$. Similar artefacts were also visible for the 12L4VF and MS250 transducers since the axial and lateral displacements were estimated using two-dimensional cross-correlations, which implies these estimations were coupled. Compared to the axial displacement and strain fields (Figure 6a-j), the lateral fields (Figure 6k-t) were noisier for all 
transducers, beam-forming strategies and line densities. In the case of two lines per pitch and Lu's-fk beam-forming as shown in Figure 6, $s_{z z}$ varied between $\pm 0.5 \%$, whereas $s_{x x}$ varied between $\pm 1 \%$.

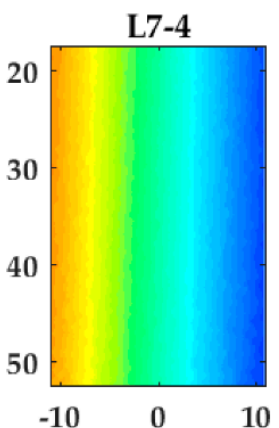

(a)

L7-4

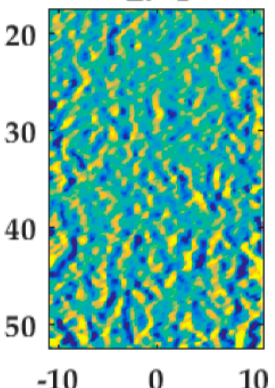

(f)

L7-4

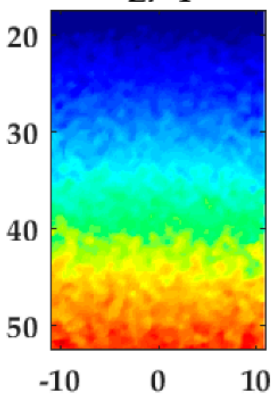

(k)

L7-4

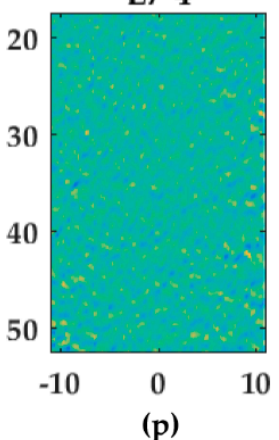

12L4VF

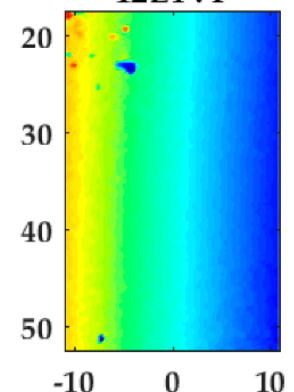

(b)

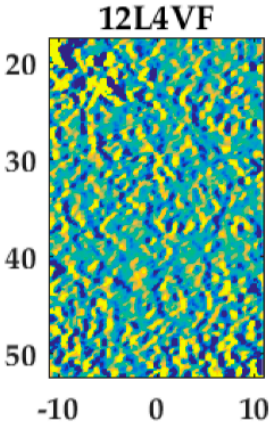

(g)

12L4VF

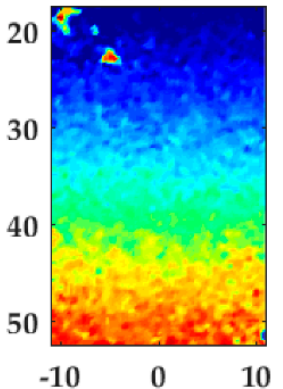

(l)

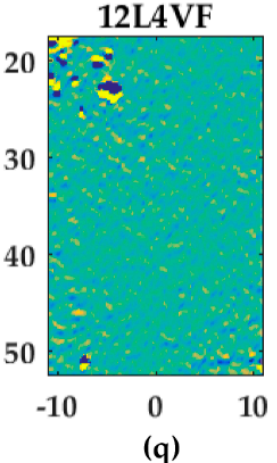

L12-5

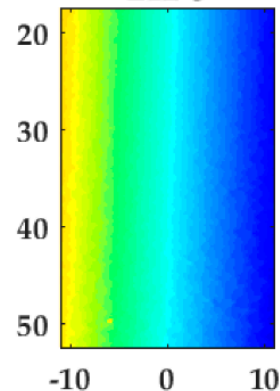

(c)

L12-5

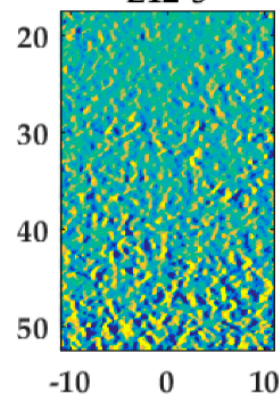

(h)

L12-5

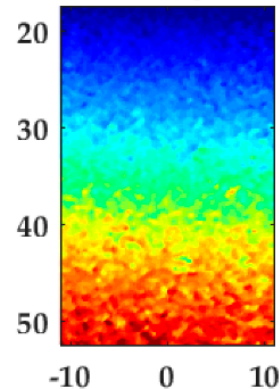

(m)

L12-5

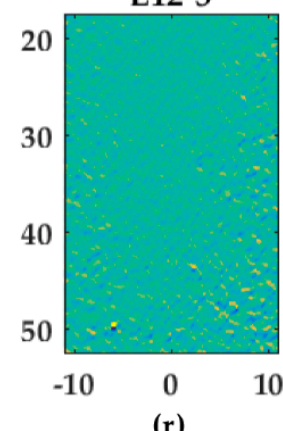

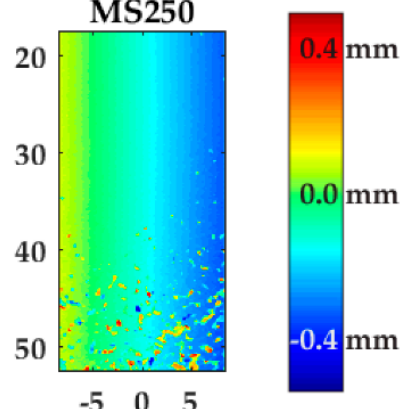

(d)

(e)

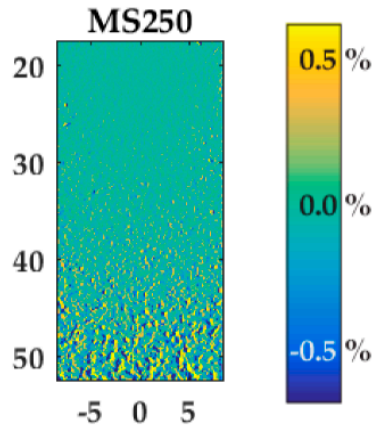

(i)

(j)

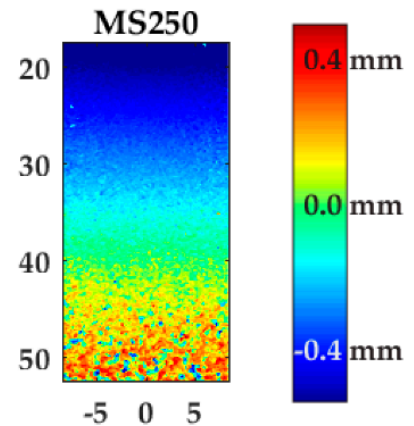

(n)

(o)

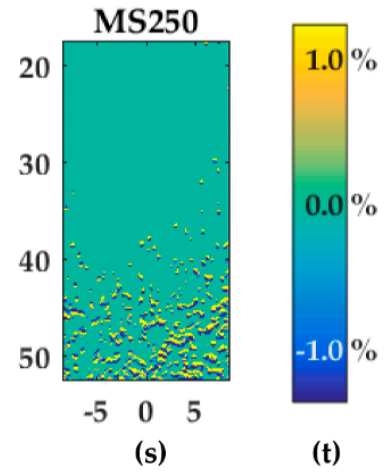

Figure 6. Overview of displacement and strain fields after rotation using Lu's-fk and two lines per pitch as a beam-forming strategy and for line density, respectively: $(\mathbf{a}-\mathbf{c})$ axial displacements; $(\mathbf{f}-\mathbf{n})$ axial strains $\left(s_{z z}\right) ;(\mathbf{k}-\mathbf{n})$ lateral displacements; $(\mathbf{p}-\mathbf{s})$ lateral strains $\left(s_{x x}\right) ;(\mathbf{e}, \mathbf{o})$ color bar in millimeters related to the figures in the same row; $(\mathbf{j}, \mathbf{t})$ color bar in percentages related to the figures in the same row. The axis of the displacement and gradient fields represents the position below the transducer in millimeters. 
In Figure 7, the performances of the axial displacement estimates are summarized for all transducers, beam-forming strategies (including angular weighting and apodization) and line densities. As can be noticed for L7-4 (Figure 7a), the RMSE seemed to be almost constant for all beam-forming strategies and line-densities except DaS with one line per pitch, in which the RMSE increased. For the 12L4VF (Figure 7b), three lines per pitch provided the lowest RMSE using Lu's-fk, Stolt's-fk or DaS, with apodization or angular weighting. Furthermore, apodization or angular weighting seemed to decrease the RMSE for this transducer. However, the opposite effect of weighting and apodization can be seen for both the L12-5 and MS250 (Figure 7c,d) in which the RMSE increased. Multiple lines seemed to slightly increase the RMSE compared to one line per pitch for all strategies in these transducers. DaS or Lu's-fk with one line per pitch resulted in the lowest RMSE in both L12-5 and MS250. The RMSE by the lateral displacements estimates (Figure 8) decreased for each method and transducer using over 1 line per pitch.

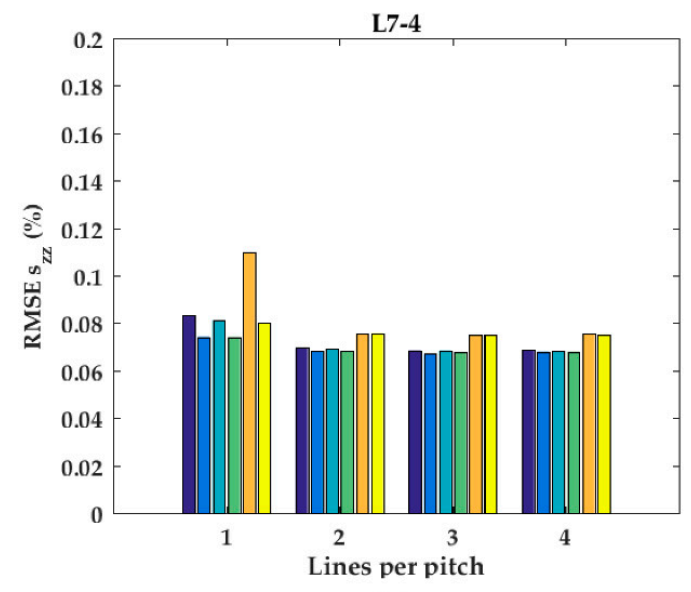

(a)

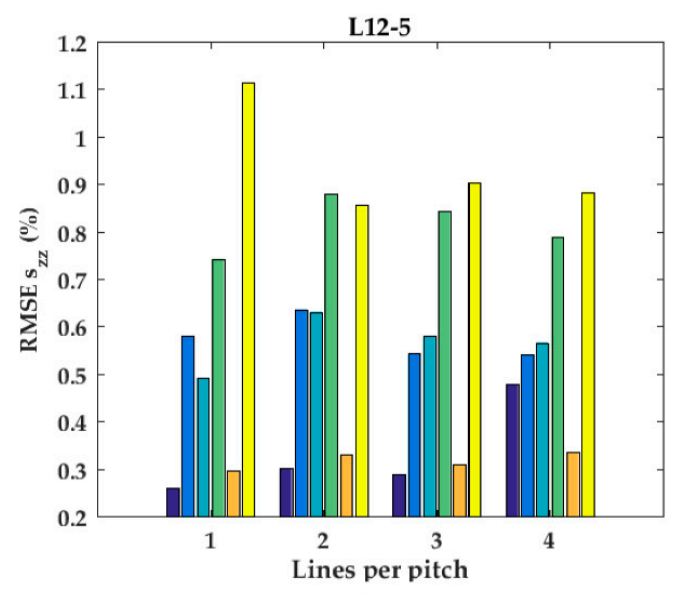

(c)

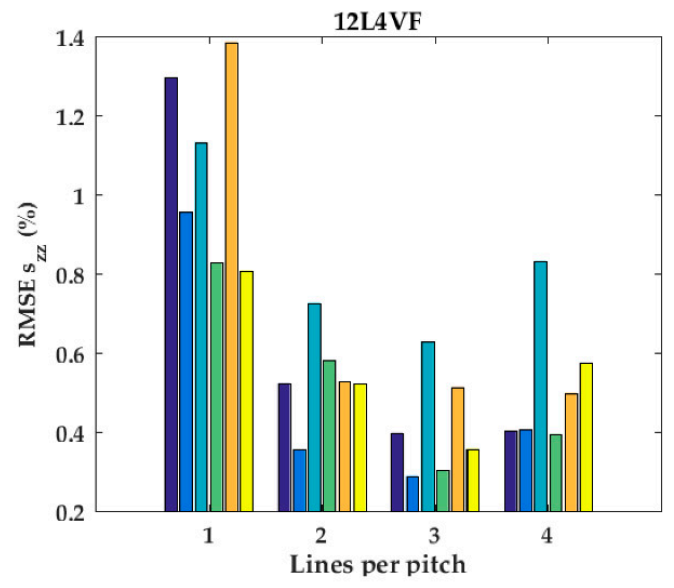

(b)

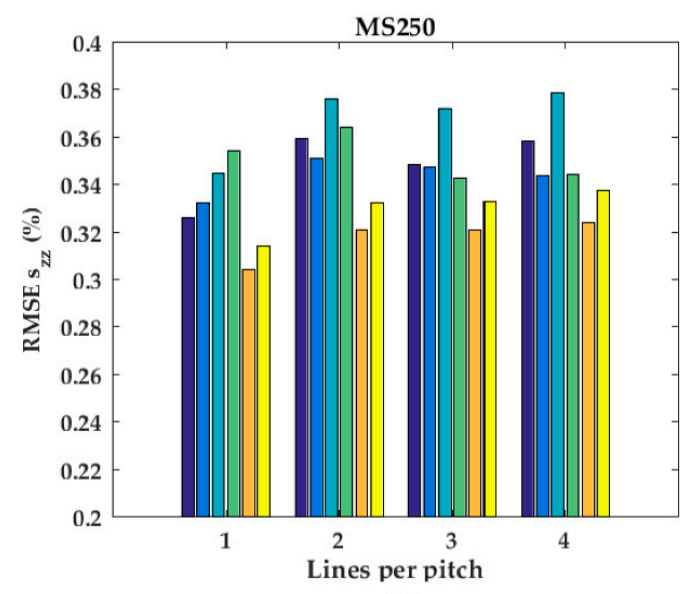

(d)

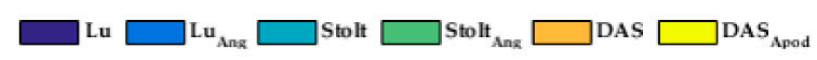

(e)

Figure 7. The root-mean-squared error (RMSE) of the axial strains $\left(s_{z z}\right)$ for all beam-forming strategies and line densities for the (a) L7-4, (b) 12L4VF, (c) L12-5, and (d) MS250 transducer; (e) is the legend used in $(\mathbf{a}-\mathbf{d})$.

The RMSE increased when applying angular weighting or apodization for all transducers except 12L4VF, for which the RMSE decreased. Lu's-fk resulted in slightly decreased RMSE compared to other methods in both L7-4 and L12-5; Lu's-fk with angular weighting in 12L4VF, and Lu's-fk and DaS 
in MS250. For the 12L4VF, the RMSEs were recalculated neglecting the left top area (squared area; axial $<28 \mathrm{~mm}$; lateral $<0 \mathrm{~mm}$, Figure $6 \mathrm{~g}, \mathrm{q}$ ) including the artifact. The results are presented in Figure 9 . Although the overall RMSE values decreased, the observed results were similar to those for the full FoV (Figures $7 \mathrm{~b}$ and $8 \mathrm{~b}$ ). Line densities of two or three lines per pitch resulted in the lowest RMSE for all strategies. Apodization or weighting seemed to decrease RMSE values but less severely and at times even increased values. This artifact might be caused by a small number of damaged elements on the left side of the transducer, resulting in artifacts especially in the near-field as only a few elements were used for reconstruction in that area.

Examples of lateral displacement and strain fields (L12-5, Lu's-fk) with a line density of one and two lines per pitch are shown in Figure 10. In the gradient field at 1 line per pitch (Figure 10b), bands with increased gradients are visible at axial positions of $20,30,40$, and $50 \mathrm{~cm}$. At these positions, the lateral displacements were approximately \pm 0.1 and $\pm 0.3 \mathrm{~mm}$, which were displacement at half-pitch positions. After beam-forming using two lines per pitch, these bands were decreased (Figure 10c,d). These bands resulted in an increased RMSE for one compared to multiple lines per pitch (Figure 10c). The appearance of these bands at sub-pitch displacements using one line per pitch were also observed for the other transducers and beam-forming methods.

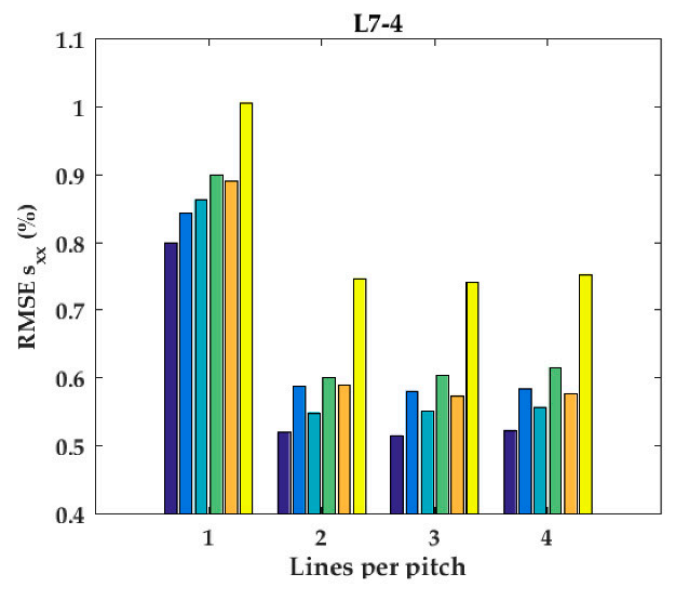

(a)

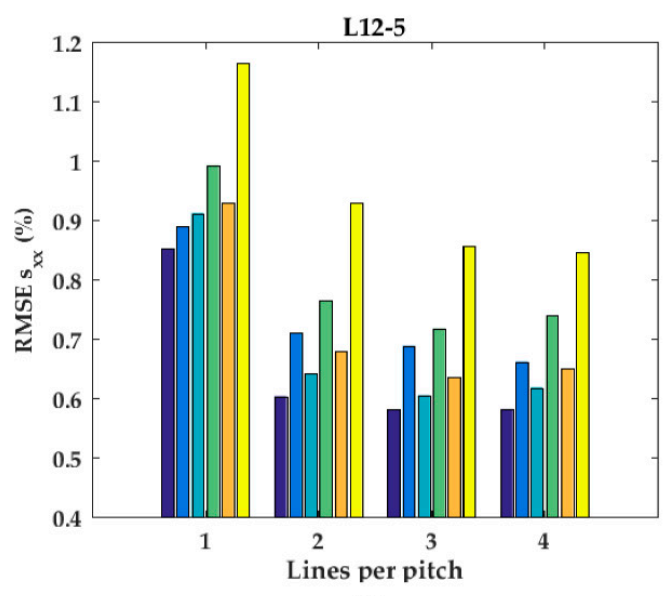

(c)

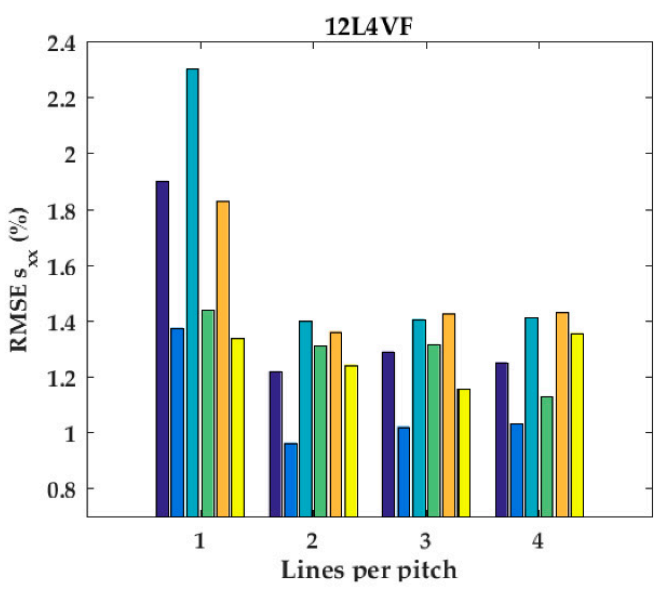

(b)

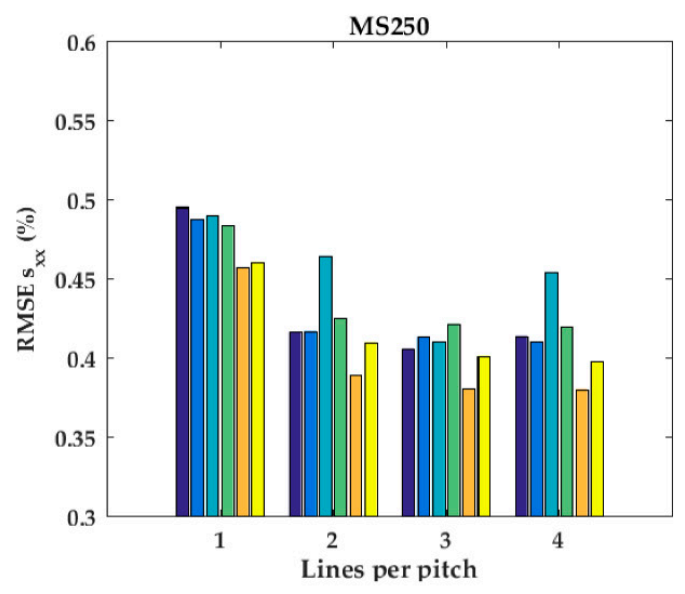

(d)

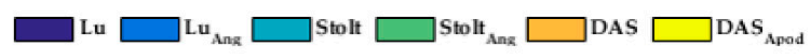

(e)

Figure 8. The root-mean-squared error (RMSE) of the lateral strains $\left(s_{x x}\right)$ for all beam-forming strategies and line densities for the (a) L7-4, (b) 12L4VF, (c) L12-5, and (d) MS250 transducer; (e) is the legend used in (a-d). 


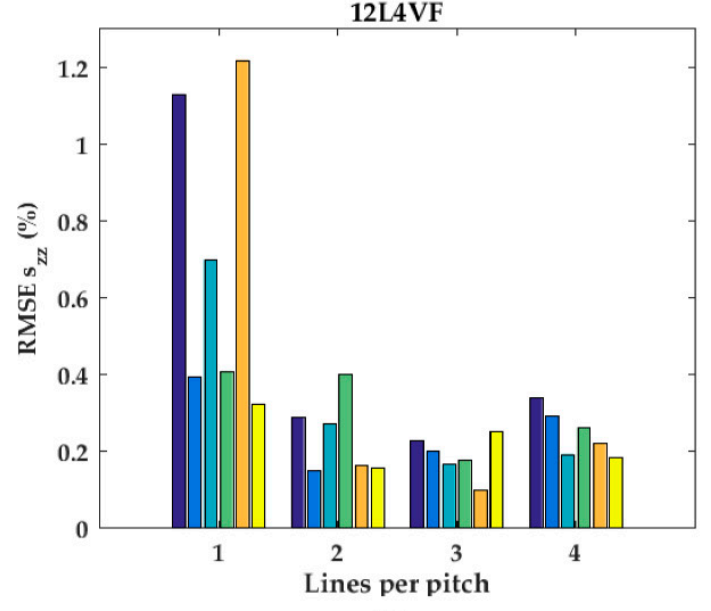

(a)

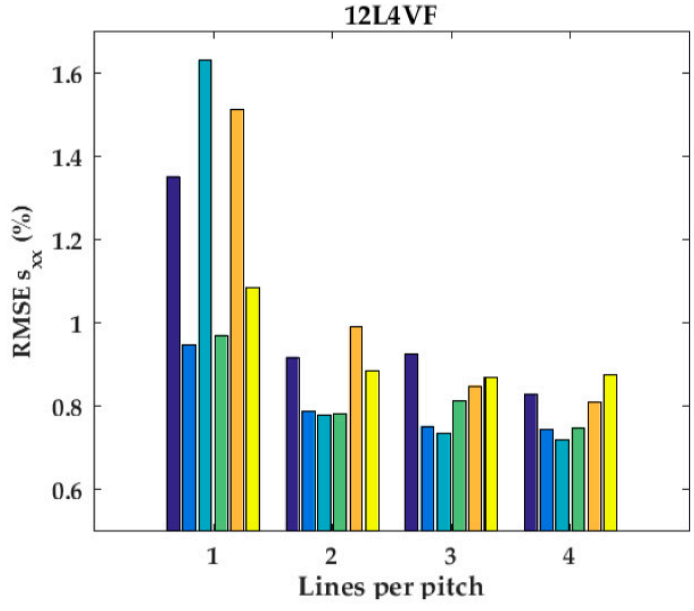

(b)

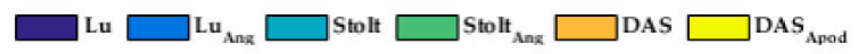

(c)

Figure 9. The root-mean-squared error (RMSE) without the top left artifact of the (a) axial strain $\left(s_{z z}\right)$ and (b) lateral strain $\left(s_{x x}\right)$ of the 12L4VF transducer; $s_{z z}$ and $s_{x x}$ values in the top left corner (axial and lateral position smaller than 28 and $0 \mathrm{~cm}$ respectively) were neglected in these RMSE calculations.

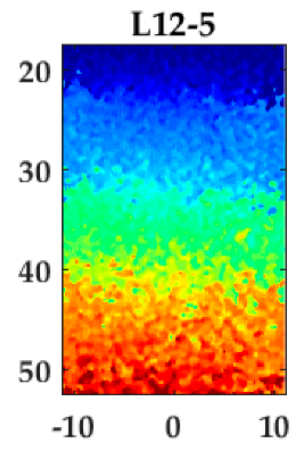

(a)

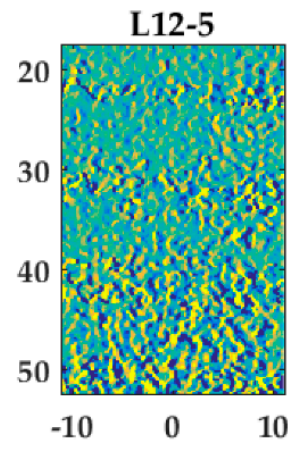

(b)

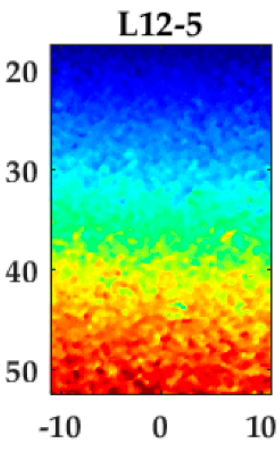

(c)

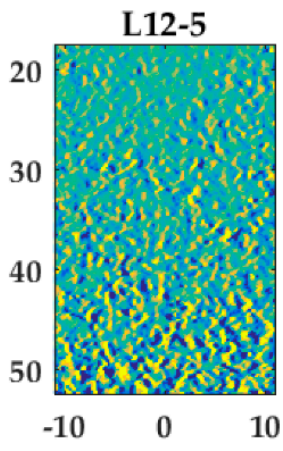

(d)

Figure 10. Lateral displacement and strain fields using Lu's-fk for the L12-5 transducer: $(\mathbf{a}, \mathbf{c})$ displacement field using one (a) or two (c) lines per pitch. Please refer to Figure 6o for the color bar; (b,d) strain field using one (b) or two (d) lines per pitch. Please refer to Figure 6j and Figure $6 \mathrm{t}$ for the corresponding color bars.

\section{Discussion}

In this study, the effect of beam-forming strategies and line density on the accuracy of normalized cross-correlation-based displacement estimation was evaluated. Therefore, we designed a method to evaluate the accuracy by phantom rotation, which had two main advantages. First, displacement fields were induced with axial and lateral displacements separately varying within $\pm 0.5 \mathrm{~mm}$ (Figure $1 \mathrm{a}-\mathrm{d}$, i-l) instead of pure axial or lateral displacements. Second, the local displacement gradients are expected to be zero independent of the rotation center and angle in this case where the rotation angles was small $\left(<<10^{\circ}\right)$. Consequently, the error of the gradient (RMSE) was used as measure for the accuracy of displacement estimates.

Displacements were calculated by normalized cross-correlation and block-matching. Although other methods are available, only this method was used in the study since it is widely used in (shear) strain imaging. The effect of different methods on the accuracy of displacements was not the scope 
of this study and so not evaluated. Furthermore, a one dimensional, three-point least-square-strain estimator (LSQSE) was implemented to calculate the gradients for evaluation. Although not presented here, equal results were obtained for LSQSE sizes of 7 and 11 points.

The accuracy of displacement estimates, especially lateral, improved significantly using a line-density of multiple lines per pitch for all transducers except the high frequency transducer MS250 in which this improvement was minimal or the accuracy even slightly decreased for some beam-forming strategies. Improvement by higher line-densities was also observed by Konofagou et al. [20] in which conventional focused ultrasound was used and RF-lines were interpolated (up to 64 lines per pitch) to gain higher line-densities to improve results. The accuracy of displacement estimates already improved by two or three lines per pitch in this study. However, interpolation of the cross-correlation peak after the final iteration was implemented in this study which also contributes to improved sub-sample displacement estimates [2]. As can be seen in Figure 10a,b, the performance of cross-correlation peak interpolation seemed to be decreased in displacements at half-pitch distances resulting in increased gradient bands (Figure 10b). This effect seemed to disappear at a line density of two lines per pitch (Figure 10c,d). The increased performance of sub-sample interpolation might be explained by the point spread function (PSF). According to Saris et al. [17], beam-forming to a PSF-based USGrid results in a circular cross-correlation peak which improves the performance of two-dimensional spline peak interpolation. The optimal number lines should be between two or three lines according to the point spread function for all transducers. This line-density matched the optimal density found in this study.

For all transducers except the 12L4VF transducer, apodization or angular weighting seemed to decrease or minimally affect the accuracy of axial and lateral displacement estimates (Figures 7 and 8). Weighting and apodization increased the lateral resolution of the beam-formed data (Appendix A), which may explain the decreased accuracy of lateral estimates. In Appendix A, the axial and lateral resolution and contrast-to-noise-ratios (CNR) of the beam-formed data by the different beam-forming, weighting and apodization methods were evaluated. The relation between lateral resolution and displacement estimation accuracy was also described previously by Luo et al. [16]. Since lateral and axial displacement estimation was coupled, the accuracy of lateral displacement estimates also affected the accuracy of the axial estimates. For the 12L4VF transducer, the opposite effect can be noticed in the results with and without the top left artifact (Figures $7 b, 8 b$ and 9). The increase in performance with apodization or angular weighting may be partially explained because weighting or apodization reduced artifacts in beam-formed data caused by broken elements in the left part of the transducer footprint. As can be observed in Figure 9, the improvement in displacement estimation accuracy by weighting was less when neglecting the artifact. Another explanation might be related to the fact that side lobes are stronger for the 12L4VF compared to the other transducers given its increased element width-to-wavelength ratio. Weighting suppresses side lobes which might also explain the improved displacement estimates despite the worse lateral resolution. In this study, a homogenous phantom was used so the effect of improved contrast (Appendix A) by weighting might be limited and has to be further investigated in an inhomogeneous phantom or in ex vivo tissue which can also be rotated. Furthermore, Lu's-fk performed equally or outperformed DaS with optimal line density

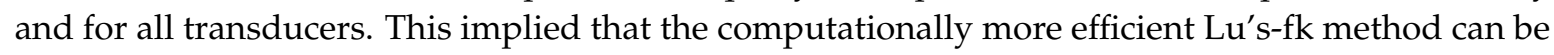
used as alternative for the widely applied DaS. Furthermore, Lu's-fk often outperformed Stolt's-fk method probably because Lu's-fk resulted in an improved lateral resolution in the beam-formed data (Appendix A).

\section{Conclusions}

In this study, we investigated the effect of different beam-forming strategies on the accuracy of displacement estimates in four different transducers (L7-4, 12L4VF, L12-5 and MS250). We developed an easy-to-implement method to evaluate the displacement estimation accuracy based on the displacement gradients estimated in a rotating phantom. A line density of multiple lines per 
pitch seemed to outperform the accuracy of the estimates compared to one line per pitch for all beam-forming strategies and transducers. Lu's-fk beam-forming seemed to outperform Stolt's-fk and resulted in similar accuracy as DaS for multiple lines per pitch. It can therefore be used interchangeable in displacement estimation without affecting accuracy, with the added advantage that the computational load is lower. In future work, an inhomogeneous phantom or ex vivo tissue might be used to improve extrapolation of results to in vivo conditions and to further investigate the effect of contrast enhancement and side lobe reduction by apodization or weighting strategies on displacement estimation.

Acknowledgments: This research is supported by Siemens Healthineers and by the Dutch Technology Foundation STW (Project 13290) which is part of the Netherlands Organization for Scientific Research (NWO) and is partly funded by the Ministry of Economic Affairs. Furthermore, the authors wish to thank Anne Saris in our group for all constructive discussions and Chi-Yin Lee, John Klepper and Andy Milkowski of Siemens Healthineers for their technical support.

Author Contributions: G.A.G.M.H. and H.H.G.H. conceived and designed the experiments; G.A.G.M.H. performed the experiments; G.A.G.M.H., C.C. and H.H.G.H. implemented the beam-forming and displacement estimation algorithms; G.A.G.M.H., C.C., H.H.G.H. and C.L.d.K. analyzed the data; G.A.G.M.H., C.C., H.H.G.H. and C.L.d.K. wrote the paper.

Conflicts of Interest: The authors declare no conflict of interest.

\section{Appendix A}

Introduction: Element data obtained by plane-wave acquisitions were beam-formed to reconstruct radio frequency $(\mathrm{RF})$ data for displacement estimation. In this study, three beam-forming strategies were used: delay-and-sum (DaS), Lu's-fk, and Stolt's-fk. In DaS beam-forming, dynamic focusing in receive was applied with and without apodization (Hamm function) in which the F-number had to be set (Equation (1)). Furthermore, angular weighting was applied in Lu's-fk and Stolt's-fk beam-forming in which the migrated k-space spectrum was multiplied with a template designed such that wave directions closer aligned to the beam-steering direction were multiplied with higher weights compared to waves deviating from the steering angle. In angular weighting, the angular range had to be set: wave directions within this range were weighted by Hann function and outside were weighted by zero. Summarized, the optimal F-number in DaS with and without apodization and the range in angular weighting were investigated.

Method and Materials: Plane-wave element data were obtained by three transducers (L7-4, 12L4VF, L12-5) in a multi-purpose phantom (model 539; ATS Laboratories, Norfolk, VA, USA) containing circular lesions and needles to evaluate contrast and resolution, respectively. Element data were beam-formed by above strategies in which the F-number $(\mathrm{DaS})$ or angular range (angular weighting) were varied between 0 and 2.0, and \pm 10 and \pm 30 . The mean contrast-to-noise ratio (CNR) of the lesions and mean axial and lateral resolution (full width at half maximum) of the needles were calculated to a depth of $50 \mathrm{~mm}$ (Figure A1) using software provided by the Plane-wave Imaging Challenge in Medical Ultrasound (PICMUS) of the IEEE International Ultrasonics Symposium 2016 [21].

Results and Discussion: In DaS, an F-number of 0.875 seemed to be most optimal for all transducers: contrast decreased while lateral resolution remained constant at values below 0.875 and contrast remained constant above 0.875 while lateral resolution increased. The axial resolution seemed to be independent of the F-number. These results were found in DaS with and without apodization. As can be seen in Table A1, apodization increased contrast, which came at the cost of increased lateral resolution.

Similar results were found for angular weighting (Table A2), a range of $\pm 20^{\circ}$ seemed to be optimal for all transducers and both Lu's-fk and Stolt's-fk, since the lateral resolution and contrast did not improve anymore at larger and smaller angle ranges, respectively. The axial resolution was unaffected by the angular range. Furthermore, angular weighting improved contrast at the cost of lateral resolution, as can be seen in comparing Tables A2 and A3. 
The multi-purpose phantom used in this study was only suitable for transducers with frequencies varying between 2 and $15 \mathrm{MHz}$. Therefore, the MS250 transducer with a center frequency of $21 \mathrm{MHz}$ could not be evaluated and the same F-number and angular range were used as for the other transducers.

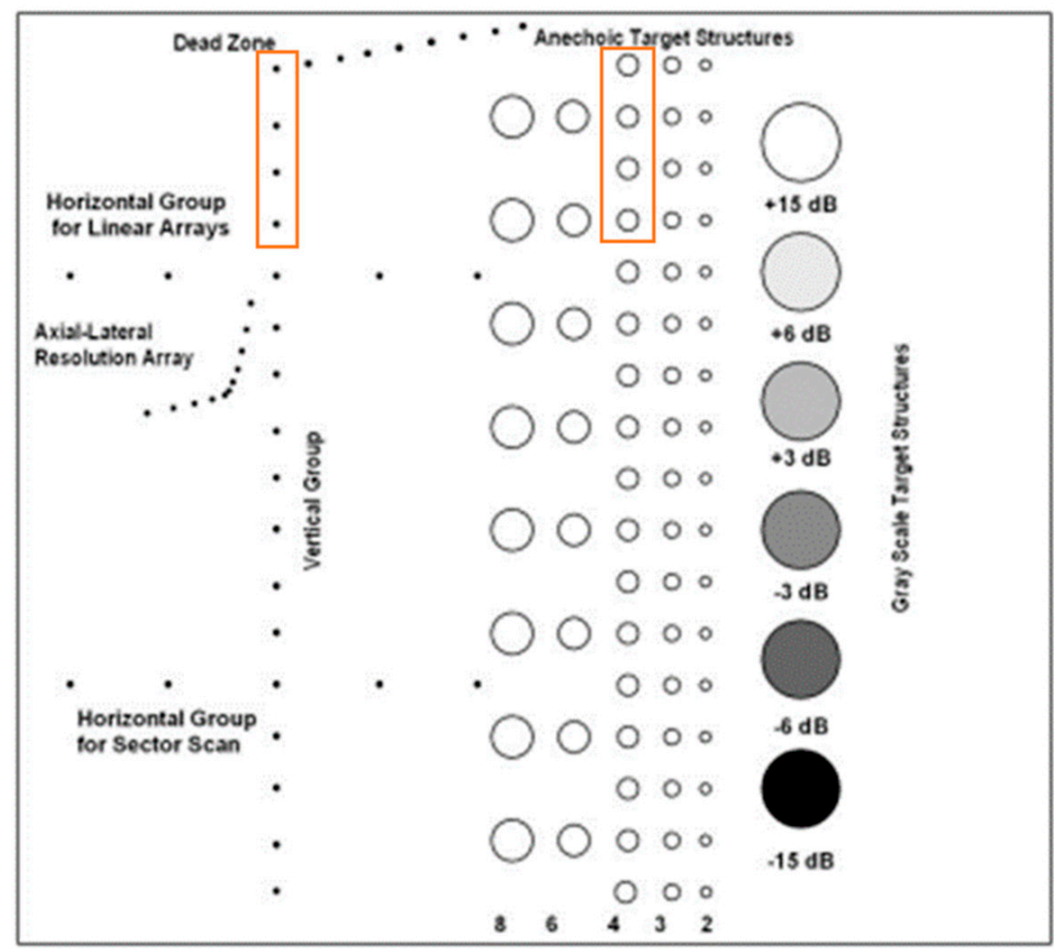

Figure A1. Cross-section of the multi-purpose phantom and the needles and lesions used for analysis indicated by the orange rectangular boxes.

Table A1. Axial and lateral resolution, and contrast-to-noise ratio (CNR) by DaS (F-number of 0.875).

\begin{tabular}{ccccc}
\hline Transducer & Apodization & Ax. Res. & Lat. Res & CNR \\
\hline L7-4 & No apod & $421 \mu \mathrm{m}$ & $490 \mu \mathrm{m}$ & $7.8 \mathrm{~dB}$ \\
- & Hamm & $422 \mu \mathrm{m}$ & $651 \mu \mathrm{m}$ & $9.7 \mathrm{~dB}$ \\
12L4VF & No apod & $422 \mu \mathrm{m}$ & $426 \mu \mathrm{m}$ & $5.4 \mathrm{~dB}$ \\
- & Hamm & $419 \mu \mathrm{m}$ & $577 \mu \mathrm{m}$ & $7.4 \mathrm{~dB}$ \\
L12-5 & No apod & $406 \mu \mathrm{m}$ & $426 \mu \mathrm{m}$ & $5.3 \mathrm{~dB}$ \\
- & Hamm & $405 \mu \mathrm{m}$ & $550 \mu \mathrm{m}$ & $7.2 \mathrm{~dB}$ \\
\hline
\end{tabular}

Table A2. Axial and lateral resolution, and CNR by angular weighted Lu's-fk and Stolt's-fk (range $\pm 20^{\circ}$ ).

\begin{tabular}{ccccc}
\hline Transducer & Method & Ax. Res. & Lat. Res & CNR \\
\hline L7-4 & Lu's-fk & $423 \mu \mathrm{m}$ & $601 \mu \mathrm{m}$ & $9.5 \mathrm{~dB}$ \\
- & Stolt's-fk & $423 \mu \mathrm{m}$ & $610 \mu \mathrm{m}$ & $9.5 \mathrm{~dB}$ \\
12L4VF & Lu's-fk & $406 \mu \mathrm{m}$ & $530 \mu \mathrm{m}$ & $6.9 \mathrm{~dB}$ \\
- & Stolt's-fk & $408 \mu \mathrm{m}$ & $616 \mu \mathrm{m}$ & $6.7 \mathrm{~dB}$ \\
L12-5 & Lu's-fk & $400 \mu \mathrm{m}$ & $510 \mu \mathrm{m}$ & $7.4 \mathrm{~dB}$ \\
- & Stolt's-fk & $401 \mu \mathrm{m}$ & $556 \mu \mathrm{m}$ & $7.4 \mathrm{~dB}$ \\
\hline
\end{tabular}


Table A3. Axial and lateral resolution, and CNR by Lu's-fk and Stolt's-fk without angular weighting.

\begin{tabular}{ccccc}
\hline Transducer & Method & Ax. Res. & Lat. Res & CNR \\
\hline L7-4 & Lu's-fk & $420 \mu \mathrm{m}$ & $493 \mu \mathrm{m}$ & $8.1 \mathrm{~dB}$ \\
& Stolt's-fk & $416 \mu \mathrm{m}$ & $507 \mu \mathrm{m}$ & $7.7 \mathrm{~dB}$ \\
12L4VF & Lu's-fk & $405 \mu \mathrm{m}$ & $423 \mu \mathrm{m}$ & $5.4 \mathrm{~dB}$ \\
& Stolt's-fk & $412 \mu \mathrm{m}$ & $584 \mu \mathrm{m}$ & $4.7 \mathrm{~dB}$ \\
L12-5 & Lu's-fk & $397 \mu \mathrm{m}$ & $420 \mu \mathrm{m}$ & $6.3 \mathrm{~dB}$ \\
& Stolt's-fk & $402 \mu \mathrm{m}$ & $532 \mu \mathrm{m}$ & $5.6 \mathrm{~dB}$ \\
\hline
\end{tabular}

\section{References}

1. Ophir, J.; Céspedes, I.; Ponnekanti, H.; Yazdi, Y.; Li, X. Elastography: A quantitative method for imaging the elasticity of biological tissues. Ultrason. Imaging 1991, 13, 111-134. [CrossRef] [PubMed]

2. Lopata, R.G.; Nillesen, M.M.; Hansen, H.H.; Gerrits, I.H.; Thijssen, J.M.; de Korte, C.L. Performance evaluation of methods for two-dimensional displacement and strain estimation using ultrasound radio frequency data. Ultrasound Med. Boil. 2009, 35, 796-812. [CrossRef] [PubMed]

3. Chen, H.; Varghese, T. Multilevel hybrid 2d strain imaging algorithm for ultrasound sector/phased arrays. Med. Phys. 2009, 36, 2098-2106. [CrossRef] [PubMed]

4. D’Hooge, J.; Heimdal, A.; Jamal, F.; Kukulski, T.; Bijnens, B.; Rademakers, F.; Hatle, L.; Suetens, P.; Sutherland, G.R. Regional strain and strain rate measurements by cardiac ultrasound: Principles, implementation and limitations. Eur. J. Echocardiogr. 2000, 1, 154-170. [CrossRef] [PubMed]

5. Luo, J.; Konofagou, E. A fast normalized cross-correlation calculation method for motion estimation. IEEE Trans. Ultrason. Ferroelectr. Freq. Control 2010, 57, 1347-1357. [PubMed]

6. Pan, X.; Gao, J.; Tao, S.; Liu, K.; Bai, J.; Luo, J. A two-step optical flow method for strain estimation in elastography: Simulation and phantom study. Ultrasonics 2014, 54, 990-996. [CrossRef] [PubMed]

7. Kallel, F.; Ophir, J. A least-squares strain estimator for elastography. Ultrason. Imaging 1997, 19, $195-208$. [CrossRef] [PubMed]

8. Delannoy, B.; Torguet, R.; Bruneel, C.; Bridoux, E. Ultrafast electronical image reconstruction device. In Echocardiology; Lancée, C.T., Ed.; Springer Netherlands: Dordrecht, The Netherlands, 1979; pp. 447-450.

9. Delannoy, B.; Torguet, R.; Bruneel, C.; Bridoux, E.; Rouvaen, J.M.; Lasota, H. Acoustical image reconstruction in parallel-processing analog electronic systems. J. Appl. Phys. 1979, 50, 3153-3159. [CrossRef]

10. Montaldo, G.; Tanter, M.; Bercoff, J.; Benech, N.; Fink, M. Coherent plane-wave compounding for very high frame rate ultrasonography and transient elastography. IEEE Trans. Ultrason. Ferroelectr. Freq. Control 2009, 56, 489-506. [CrossRef] [PubMed]

11. Lu, J.Y. 2D and 3D high frame rate imaging with limited diffraction beams. IEEE Trans. Ultrason. Ferroelectr. Freq. Control 1997, 44, 839-856. [CrossRef]

12. Lu, J.Y. Experimental study of high frame rate imaging with limited diffraction beams. IEEE Trans. Ultrason. Ferroelectr. Freq. Control 1998, 45, 84-97. [CrossRef] [PubMed]

13. Garcia, D.; Le Tarnec, L.; Muth, S.; Montagnon, E.; Poree, J.; Cloutier, G. Stolt's f-k migration for plane wave ultrasound imaging. IEEE Trans. Ultrason. Ferroelectr. Freq. Control 2013, 60, 1853-1867. [CrossRef] [PubMed]

14. Chen, C.; Hendriks, G.A.G.M.; Hansen, H.H.G.; de Korte, C.L. Design of an angular weighting template for coherent plane wave compounding in fourier domain. In Proceedings of the 2017 IEEE International Ultrasonics Symposium (IUS), Washington, DC, USA, 7-9 September 2017; p. 1.

15. Ophir, J.; Alam, S.K.; Garra, B.; Kallel, F.; Konofagou, E.; Krouskop, T.; Varghese, T. Elastography: Ultrasonic estimation and imaging of the elastic properties of tissues. Proc. Inst. Mech. Eng. Part H J. Eng. Med. 1999, 213, 203-233. [CrossRef] [PubMed]

16. Luo, J.; Konofagou, E.E. Effects of various parameters on lateral displacement estimation in ultrasound elastography. Ultrasound Med. Boil. 2009, 35, 1352-1366. [CrossRef] [PubMed]

17. Saris, A.E.C.M.; Nillesen, M.M.; Fekkes, S.; Hansen, H.H.G.; de Korte, C.L. Robust blood velocity estimation using point-spread-function-based beamforming and multi-step speckle tracking. In Proceedings of the 2015 IEEE International Ultrasonics Symposium (IUS), Taipei, Taiwan, 21-24 October 2015; pp. 1-4. 
18. Hansen, H.H.G.; Lopata, R.G.P.; Idzenga, T.; de Korte, C.L. Full 2D displacement vector and strain tensor estimation for superficial tissue using beam-steered ultrasound imaging. Phys. Med. Boil. 2010, 55, 3201-3218. [CrossRef] [PubMed]

19. Fekkes, S.; Swillens, A.E.; Hansen, H.H.; Saris, A.E.; Nillesen, M.M.; Iannaccone, F.; Segers, P.; de Korte, C.L. $2 \mathrm{D}$ versus $3 \mathrm{D}$ cross-correlation-based radial and circumferential strain estimation using multiplane $2 \mathrm{~d}$ ultrafast ultrasound in a 3d atherosclerotic carotid artery model. IEEE Trans. Ultrason. Ferroelectr. Freq. Control 2016, 63, 1543-1553. [CrossRef] [PubMed]

20. Konofagou, E.; Ophir, J. A new elastographic method for estimation and imaging of lateral displacements, lateral strains, corrected axial strains and poisson's ratios in tissues. Ultrasound Med. Biol. 1998, 24, 1183-1199. [CrossRef]

21. Liebgott, H.; Rodriguez-Molares, A.; Cervenansky, F.; Jensen, J.A.; Bernard, O. Plane-wave imaging challenge in medical ultrasound. In Proceedings of the 2016 IEEE International Ultrasonics Symposium (IUS), Tours, France, 18-21 September 2016; pp. 1-4.

2018 by the authors. Licensee MDPI, Basel, Switzerland. This article is an open access article distributed under the terms and conditions of the Creative Commons Attribution (CC BY) license (http:// creativecommons.org/licenses/by/4.0/). 